\section{Home in hyperspace}

\section{Deborah Singmaster}

The Fourth Dimension and How to

Get There. *

By Rudy Rucker.

Rider-Hutchinson: 1985. Pp.228.

£9.95.

THE dust jacket of the British edition of Rudy Rucker's The Fourth Dimension carries a picture that looks like a shot from the final sequence of Star Wars. It could be a post card from the fourth dimension, and if it were from the author it would say "Wish you were here!". Rucker admits that he is in love with cosmology and in this exhilarating book he tries to convey his enthusiasm to his readers.

The book is dedicated to "A Square, on his hundredth anniversary" - an apt tribute to another book on the same theme, Flatland by the English school master Edwin A. Abbott, published in 1884 and gallantly kept in print in Britain by Basil Blackwell. It deserves to be more widely read than it is. Rucker makes frequent references to Flatland throughout his book; he has even written a sequel to the original.

Starting with Plato and the allegory of the shadows on the wall of the cave, Rucker moves by analogy towards a description of a spatial fourth dimension. He introduces the hypercube, "probably the best-known 4-D geometrical problem", and proceeds to the hypersphere, hyperspace tunnels, the shape of space, spacetime and so on. From these literal interpretations of a fourth dimension, he passes to more abstract uses of the term and suggests that we, with our complex interior world of thoughts, feelings, dreams, are "in some very real sense, beings of more than three dimensions".

Rucker has no difficulty in thinking four dimensionally. He quotes Charles Hinton, "the great hyperspace philosopher" and a contemporary of Abbott's, as saying that one of the best methods of preparing oneself for four-dimensional thought is to create a deliberate confusion between left and right, by experimenting with mirror images, for example. One of the most entertaining of the many anecdotes in the book is Rucker's potted biography of Hinton, the son of James Hinton who advocated and practised free love. Charles, who was himself convicted of bigamy, believed that "space is, at the deepest level, to be known by the heart and not the brain"'. But that way mysticism lies: Rucker is at times unashamedly mystical.

For mathematical greenhorns, Rucker provides simple explanations of major concepts including Einstein's theory of

* In the United States The Fourth Dimension: Toward A Geometry of Higher Reality, published by Houghton Mifflin. A technical review of the book appeared in Nature 312, 214 (1984). relativity, quantum mechanics and David Hilbert's theory of infinite dimensional spaces. He also discusses less orthodox notions such as synchronicity and the possibility of time travel - he is, after all, apart from being a mathematical logician, a writer of science fiction.

Appropriately, The Fourth Dimension is a fourfold book. Besides the text there are witty and helpful diagrams and illustrations by David Povilaitis, the margins contain extensive quotations from writers as diverse as Plato, Ouspensky, Lewis Carroll and Borges, and there are also puzzles for those whom Martin Gardner, in his foreword, describes as "the

\section{Distant reactions}

Alexander Dalgarno

Interstellar Chemistry.

By W.W. Duley and D.A. Williams. Academic:1984. Pp.251. \$45, £29.

THE discovery in the dense regions of interstellar space of molecules, ranging in complexity from simple diatomic species to extended linear chain polyynes, has provided a unique diagnostic probe into the physical conditions in which star formation occurs. Analysis of the intensities of molecular emission and absorption lines yields estimates of the densities, temperatures, velocities and radiation characteristics of the environment; observations of absorption by rotational levels of the diatomic cyanogen molecule $\mathrm{CN}$ have given us a measure of the temperature of the blackbody radiation of the Universe at wavelengths of $2.6 \mathrm{~mm}$ and $1.3 \mathrm{~mm}$; and observations of emission from deuterated molecules have yielded a value for the density of matter in the early Universe, showing that baryonic matter cannot close the Universe.

Molecules are found also in the material ejected by protostellar objects as they begin their evolution to the main sequence, and in the circumstellar shells and stellar winds of massive stars which undergo mass loss throughout their existence. Molecular masers are clear signposts marking phases in the sequence of events that leads to the formation of stars and the dissipation of the remnant material out of which they are made. Vibrationally excited molecular hydrogen is widely observed and provides insight into a diverse range of phenomena, among them galaxies in collision.

Molecules are much more than probes of exotic environments, however; they are also highly effective cooling agents. Molecular emissions carry away thermal energy and bring molecular clouds to the brink of gravitational collapse. Molecules play a controlling role in the ionization balance of collapsing objects and in the dissipation of rotational angular momentum by magnetic braking. mathematically competent"'. (Answers are supplied.)

Rucker's book is not aimed at the trained mathematician or physicist but it should appeal to anyone else who is interested in the nature of the Universe and our part in it. Science undergraduates, even sixth formers, and all science fiction enthusiasts will find it (and Flatland) stimulating reading. This is a refreshing and thoughtprovoking book written by an engaging optimist for whom, unlike Pascal, infinity holds no fears.

Deborah Singmaster is a freelance writer and broadcaster.

To utilize fully molecular observations in the investigation of astronomical phenomena we must understand the nature of the processes by which molecules are formed and destroyed. Those processes belong to a special kind of chemistry, a chemistry that is now an integral part of astronomy and that is the subject of Duley and Williams's book.

Interstellar chemistry is a combination of gas phase chemistry and surface chemistry. The phenomena under study occur at densities too low for the threebody processes that control terrestrial gas phase chemistry, and the formation of molecular compounds is initiated by radiative associations in which the reactions are stabilized by the emission of photons. The temperatures can be as low as $5 \mathrm{~K}$, though in localized regions the passage of a shock heats the gas to several thousand degrees and may dissociate the molecules which then form into new compounds as the heated gas cools and the atoms recombine. The interstellar medium contains grains, refractory particles of dimensions of the order of $10^{-5} \mathrm{~cm}$, on whose surfaces molecules may form to be later released into the gas phase. The gas phase and surface reactions take place together in a medium bombarded by energetic cosmic rays and subjected to X-rays and to ultraviolet photons from embedded stars and exploding supernovae.

Thus a diverse array of basic chemical processes forms the content of interstellar chemistry. Duley and Williams have written a lucid account of these processes in a book in which the background is developed sufficiently to make apparent the relevance of chemistry to astronomy. Here the two disciplines are woven together in a pattern of absorbing interest which should appeal to a broad range of scientists. A limited familiarity with elementary molecular physics is adequate preparation, but at the same time the authoritative tone reflects the fact that both authors are active researchers in this field. Their book will come to be seen as the standard introduction to the subject.

Alexander Dalgarno is Phillips Professor of Astronomy at Harvard University. 